\title{
COMPOUND INVERSIVE CONGRUENTIAL PSEUDORANDOM NUMBERS: AN AVERAGE-CASE ANALYSIS
}

\author{
JÜRGEN EICHENAUER-HERRMANN AND FRANK EMMERICH
}

\begin{abstract}
The present paper deals with the compound (or generalized) inversive congruential method for generating uniform pseudorandom numbers, which has been introduced recently. Equidistribution and statistical independence properties of the generated sequences over parts of the period are studied based on the discrepancy of certain point sets. The main result is an upper bound for the average value of these discrepancies. The method of proof is based on estimates for exponential sums.
\end{abstract}

\section{INTRODUCTION}

Several nonlinear methods of generating uniform pseudorandom numbers in the interval $[0,1)$ have been introduced and studied during the last years. The development of this attractive field of research is described in the survey articles $[2,5,12,13,14]$ and in Niederreiter's excellent monograph [15]. A particularly promising approach is the inversive congruential method. The generated sequences of pseudorandom numbers have nice equidistribution and statistical independence properties (cf. $[3,10,11]$ ). Recently, a compound (or generalized) version of this method was introduced and analyzed (cf. $[4,8]$ ), which shows some additional computational advantages. The present paper deals with the average behavior of these compound inversive congruential pseudorandom numbers and includes corresponding new results for the (ordinary) inversive congruential method.

Let $p_{1}, \ldots, p_{r} \geq 5$ be distinct primes. For $1 \leq i \leq r$ identify $\mathbb{Z}_{p_{i}}=\{0,1, \ldots$, $\left.p_{i}-1\right\}$ with the finite field of order $p_{i}$. Let $a_{i} \in \mathbb{Z}_{p_{i}}^{*}=\mathbb{Z}_{p_{i}} \backslash\{0\}$ and let $\left(z_{n}^{(i)}\right)_{n \geq 0}$ be a sequence in $\mathbb{Z}_{p_{i}}$ with

$$
z_{n+1}^{(i)} \equiv a_{i}\left(z_{n}^{(i)}\right)^{-1}+1\left(\bmod p_{i}\right), \quad n \geq 0,
$$

where $z^{-1}$ denotes the multiplicative inverse of $z$ in $\mathbb{Z}_{p_{i}}^{*}$ and $0^{-1}=0$. Obviously, the sequence $\left(z_{n}^{(i)}\right)_{n \geq 0}$ is always purely periodic and $p_{i}$ is the maximum possible period length. Let $\mathbb{M}_{p_{i}}$ be the set of all $a_{i} \in \mathbb{Z}_{p_{i}}^{*}$ which belong to sequences with period length $p_{i}$. The set $\mathbb{M}_{p_{i}}$ is always nonvoid and its elements can be characterized by properties of the polynomial $x^{2}-x-a_{i} \in \mathbb{Z}_{p_{i}}[x]$ (cf. [7]). In the following, let $a_{i} \in \mathbb{M}_{p_{i}}$ and $c_{i} \in \mathbb{Z}_{p_{i}}^{*}$. Let $\left(y_{n}^{(i)}\right)_{n \geq 0}$ with $y_{0}^{(i)} \equiv c_{i} z_{0}^{(i)}\left(\bmod p_{i}\right)$ and

$$
y_{n+1}^{(i)} \equiv a_{i} c_{i}^{2}\left(y_{n}^{(i)}\right)^{-1}+c_{i}\left(\bmod p_{i}\right), \quad n \geq 0,
$$

Received by the editor September 19, 1994.

1991 Mathematics Subject Classification. Primary 65C10; Secondary 11K45.

Key words and phrases. Uniform pseudorandom numbers, compound inversive congruential method, equidistribution, statistical independence, discrepancy, exponential sums.

(C)1996 American Mathematical Society 
be the corresponding (ordinary) inversive congruential sequence of elements of $\mathbb{Z}_{p_{i}}$, and let $\left(x_{n}^{(i)}\right)_{n \geq 0}$ with

$$
x_{n}^{(i)}=y_{n}^{(i)} / p_{i} \in[0,1), \quad n \geq 0,
$$

be the corresponding stream of (ordinary) inversive congruential pseudorandom numbers. A short calculation shows that

$$
y_{n}^{(i)} \equiv c_{i} z_{n}^{(i)}\left(\bmod p_{i}\right)
$$

for any $n \geq 0$. Now, a sequence $\left(x_{n}\right)_{n \geq 0}$ of compound inversive congruential pseudorandom numbers in the interval $[0,1)$ is defined by

$$
x_{n} \equiv x_{n}^{(1)}+\cdots+x_{n}^{(r)}(\bmod 1), \quad n \geq 0 .
$$

Since the primes $p_{1}, \ldots, p_{r}$ are distinct, the sequence $\left(x_{n}\right)_{n \geq 0}$ is purely periodic with period length $m=p_{1} \cdots p_{r}$ and $x_{0}, x_{1}, \ldots, x_{m-1}$ runs through all rationals in $[0,1)$ with denominator $m$. It should be observed that in the compound inversive congruential method a very large period length $m$ can be obtained, although exact integer computations have to be performed only in $\mathbb{Z}_{p_{1}}, \ldots, \mathbb{Z}_{p_{r}}$. Additionally, the compound approach is particularly suitable for parallelized computations, since the computation of the underlying sequences $\left(x_{n}^{(i)}\right)_{n \geq 0}$ of (ordinary) inversive congruential pseudorandom numbers can be allocated to $r$ parallel processors.

Equidistribution and statistical independence properties of the generated sequences, which are very important for their usability in a stochastic simulation, can be analyzed based on the discrepancy of $s$-tuples of successive pseudorandom numbers with $s=1$ and $s \geq 2$, respectively. For $N$ arbitrary points $\mathbf{t}_{0}, \mathbf{t}_{1}, \ldots, \mathbf{t}_{N-1} \in$ $[0,1)^{s}$ the discrepancy is defined by

$$
D_{N}\left(\mathbf{t}_{0}, \mathbf{t}_{1}, \ldots, \mathbf{t}_{N-1}\right)=\sup _{J}\left|F_{N}(J)-V(J)\right|,
$$

where the supremum is extended over all subintervals $J$ of $[0,1)^{s}, F_{N}(J)$ is $N^{-1}$ times the number of points among $\mathbf{t}_{0}, \mathbf{t}_{1}, \ldots, \mathbf{t}_{N-1}$ falling into $J$, and $V(J)$ denotes the $s$-dimensional volume of $J$. In the following, nonoverlapping $s$-tuples

$$
\mathbf{x}_{n}=\left(x_{s n}, x_{s n+1}, \ldots, x_{s n+s-1}\right) \in[0,1)^{s}, \quad n \geq 0,
$$

of compound inversive congruential pseudorandom numbers are considered, and the abbreviation

$$
D_{N ; c_{1}, \ldots, c_{r}}^{(s)}=D_{N}\left(\mathbf{x}_{0}, \mathbf{x}_{1}, \ldots, \mathbf{x}_{N-1}\right)
$$

is used for $1 \leq N \leq m$. The main result of the present paper is established in the third section, namely an upper bound for the average value of the discrepancy $D_{N ; c_{1}, \ldots, c_{r}}^{(s)}$ over the parameters $c_{1}, \ldots, c_{r}$. A detailed discussion of this result is given in the fourth section. The second section contains necessary auxiliary results.

\section{Auxiliary Results}

First, some further notation is necessary. For integers $k \geq 1$ and $q \geq 2$ let $C_{k}(q)$ be the set of all nonzero lattice points $\left(h_{1}, \ldots, h_{k}\right) \in \mathbb{Z}^{k}$ with $-q / 2<h_{j} \leq q / 2$ for 
$1 \leq j \leq k$. Define

$$
r(h, q)= \begin{cases}q \sin (\pi|h| / q) & \text { for } h \in C_{1}(q) \\ 1 & \text { for } h=0\end{cases}
$$

and

$$
r(\mathbf{h}, q)=\prod_{j=1}^{k} r\left(h_{j}, q\right)
$$

for $\mathbf{h}=\left(h_{1}, \ldots, h_{k}\right) \in C_{k}(q)$. For real $t$ the abbreviation $e(t)=e^{2 \pi i t}$ is used, and $\mathbf{u} \cdot \mathbf{v}$ stands for the standard inner product of $\mathbf{u}, \mathbf{v} \in \mathbb{R}^{k}$. Subsequently, three known general results are stated which follow from [15, Theorem 3.10 and Corollary 3.17] and [6, Lemma 3], respectively.

Lemma 1. Let $N \geq 1$ and $q \geq 2$ be integers. Let $\mathbf{t}_{n}=\mathbf{y}_{n} / q \in[0,1)^{k}$ with $\mathbf{y}_{n} \in$ $\{0,1, \ldots, q-1\}^{k}$ for $0 \leq n<N$. Then the discrepancy of the points $\mathbf{t}_{0}, \mathbf{t}_{1}, \ldots, \mathbf{t}_{N-1}$ satisfies

$$
D_{N}\left(\mathbf{t}_{0}, \mathbf{t}_{1}, \ldots, \mathbf{t}_{N-1}\right) \leq \frac{k}{q}+\frac{1}{N} \sum_{\mathbf{h} \in C_{k}(q)} \frac{1}{r(\mathbf{h}, q)}\left|\sum_{n=0}^{N-1} e\left(\mathbf{h} \cdot \mathbf{t}_{n}\right)\right|
$$

Lemma 2. The discrepancy of $N$ arbitrary points $\mathbf{t}_{0}, \mathbf{t}_{1}, \ldots, \mathbf{t}_{N-1} \in[0,1)^{k}$ satisfies

$$
D_{N}\left(\mathbf{t}_{0}, \mathbf{t}_{1}, \ldots, \mathbf{t}_{N-1}\right) \geq \frac{\pi}{2 N\left((\pi+1)^{l}-1\right) \prod_{j=1}^{k} \max \left(1,\left|h_{j}\right|\right)}\left|\sum_{n=0}^{N-1} e\left(\mathbf{h} \cdot \mathbf{t}_{n}\right)\right|
$$

for any nonzero lattice point $\mathbf{h}=\left(h_{1}, \ldots, h_{k}\right) \in \mathbb{Z}^{k}$, where $l$ denotes the number of nonzero coordinates of $\mathbf{h}$.

Lemma 3. Let $q \geq 2$ be an integer. Then

$$
\sum_{\substack{\mathbf{h} \in C_{k}(q) \\ \mathbf{h} \equiv \mathbf{0}(\bmod d)}} \frac{1}{r(\mathbf{h}, q)}<\frac{1}{d}\left(\frac{2}{\pi} \log q+\frac{7}{5}\right)^{k}
$$

for any divisor $d$ of $q$ with $1 \leq d<q$.

Subsequently, the $s$-tuples

$$
\mathbf{z}_{n}^{(i)}=\left(z_{s n}^{(i)}, z_{s n+1}^{(i)}, \ldots, z_{s n+s-1}^{(i)}\right) \in \mathbb{Z}_{p_{i}}^{s}
$$

for $1 \leq i \leq r$ and $n \geq 0$ play a crucial role for the analysis of the discrepancy $D_{N ; c_{1}, \ldots, c_{r}}^{(s)}$.

Lemma 4. Let $1 \leq i \leq r, 1 \leq s<p_{i}, h_{0} \in \mathbb{Z}$, and $\mathbf{h} \in \mathbb{Z}^{s}$ with $\mathbf{h} \not \equiv \mathbf{0}\left(\bmod p_{i}\right)$. Then

$$
\#\left\{0 \leq n<p_{i} \mid \mathbf{h} \cdot \mathbf{z}_{n}^{(i)} \equiv h_{0}\left(\bmod p_{i}\right)\right\} \leq 2 s-1
$$

Proof. Let $\mathbf{h}=\left(h_{1}, \ldots, h_{s}\right)$. Since the sequence $\left(z_{n}^{(i)}\right)_{n \geq 0}$ has period length $p_{i}$, one obtains 


$$
\begin{aligned}
\#\{0 & \left.\leq n<p_{i} \mid \mathbf{h} \cdot \mathbf{z}_{n}^{(i)} \equiv h_{0}\left(\bmod p_{i}\right)\right\} \\
& =\#\left\{0 \leq n<p_{i} \mid h_{1} z_{s n}^{(i)}+\cdots+h_{s} z_{s n+s-1}^{(i)} \equiv h_{0}\left(\bmod p_{i}\right)\right\} \\
& =\#\left\{0 \leq n<p_{i} \mid h_{1} z_{n}^{(i)}+\cdots+h_{s} z_{n+s-1}^{(i)} \equiv h_{0}\left(\bmod p_{i}\right)\right\} \\
& \leq s-1+\#\left\{0 \leq n<p_{i} \mid z_{n}^{(i)} \cdots z_{n+s-2}^{(i)} \neq 0,\right. \\
& \left.\quad h_{1} z_{n}^{(i)}+\cdots+h_{s} z_{n+s-1}^{(i)} \equiv h_{0}\left(\bmod p_{i}\right)\right\} \\
& \leq 2 s-1,
\end{aligned}
$$

where the last inequality follows from [1, Theorem], [15, Theorem 8.6] which says that the hyperplane

$$
H=\left\{\left(z_{1}, \ldots, z_{s}\right) \in \mathbb{Z}_{p_{i}}^{s} \mid h_{1} z_{1}+\cdots+h_{s} z_{s} \equiv h_{0}\left(\bmod p_{i}\right)\right\}
$$

contains at most $s$ of the points $\left(z_{n}^{(i)}, \ldots, z_{n+s-1}^{(i)}\right)$ with $z_{n}^{(i)} \cdots z_{n+s-2}^{(i)} \neq 0$ and $0 \leq n<p_{i}$.

In the following, let $m_{I}=\prod_{i \in I} p_{i}$ for subsets $I$ of $\{1, \ldots, r\}$.

Lemma 5. Let $1 \leq s<\min \left\{p_{1}, \ldots, p_{r}\right\}, 1 \leq N \leq m, \mathbf{h} \in C_{s}(m)$, and $J=$ $\left\{1 \leq i \leq r \mid \mathbf{h} \equiv \mathbf{0}\left(\bmod p_{i}\right)\right\}$. Then

$$
\sum_{\left(c_{1}, \ldots, c_{r}\right) \in \mathbb{Z}_{p_{1}}^{*} \times \cdots \times \mathbb{Z}_{p_{r}}^{*}}\left|\sum_{n=0}^{N-1} e\left(\sum_{i=1}^{r} c_{i}\left(\mathbf{h} \cdot \mathbf{z}_{n}^{(i)}\right) / p_{i}\right)\right|^{2} \leq N m_{J}^{2} \prod_{\substack{i=1 \\ i \notin J}}^{r}\left(2 s\left(p_{i}-1\right)+1\right) .
$$

Proof. Straightforward calculations show that

$$
\begin{aligned}
& \sum_{\left(c_{1}, \ldots, c_{r}\right) \in \mathbb{Z}_{p_{1}}^{*} \times \cdots \times \mathbb{Z}_{p_{r}}^{*}}\left|\sum_{n=0}^{N-1} e\left(\sum_{i=1}^{r} c_{i}\left(\mathbf{h} \cdot \mathbf{z}_{n}^{(i)}\right) / p_{i}\right)\right|^{2} \\
= & \sum_{\left(c_{1}, \ldots, c_{r}\right) \in \mathbb{Z}_{p_{1}}^{*} \times \cdots \times \mathbb{Z}_{p_{r}}^{*}} \sum_{k, n=0}^{N-1} e\left(\sum_{i=1}^{r} c_{i}\left(\mathbf{h} \cdot \mathbf{z}_{n}^{(i)}-\mathbf{h} \cdot \mathbf{z}_{k}^{(i)}\right) / p_{i}\right) \\
= & \sum_{k, n=0}^{N-1} \sum_{\left(c_{1}, \ldots, c_{r}\right) \in \mathbb{Z}_{p_{1}}^{*} \times \cdots \times \mathbb{Z}_{p_{r}}^{*}} \prod_{i=1}^{r} e\left(c_{i}\left(\mathbf{h} \cdot \mathbf{z}_{n}^{(i)}-\mathbf{h} \cdot \mathbf{z}_{k}^{(i)}\right) / p_{i}\right) \\
= & \sum_{k, n=0}^{N-1} \prod_{i=1}^{r} \sum_{c \in \mathbb{Z}_{p_{i}}^{*}} e\left(c\left(\mathbf{h} \cdot \mathbf{z}_{n}^{(i)}-\mathbf{h} \cdot \mathbf{z}_{k}^{(i)}\right) / p_{i}\right) .
\end{aligned}
$$

Since 


$$
\sum_{c \in \mathbb{Z}_{p_{i}}^{*}} e\left(c\left(\mathbf{h} \cdot \mathbf{z}_{n}^{(i)}-\mathbf{h} \cdot \mathbf{z}_{k}^{(i)}\right) / p_{i}\right)= \begin{cases}p_{i}-1 & \text { for } \mathbf{h} \cdot \mathbf{z}_{n}^{(i)} \equiv \mathbf{h} \cdot \mathbf{z}_{k}^{(i)}\left(\bmod p_{i}\right), \\ -1 & \text { for } \mathbf{h} \cdot \mathbf{z}_{n}^{(i)} \not \equiv \mathbf{h} \cdot \mathbf{z}_{k}^{(i)}\left(\bmod p_{i}\right)\end{cases}
$$

for $1 \leq i \leq r$ and $0 \leq k, n<N$, it follows that

$$
\begin{aligned}
& \sum_{\left(c_{1}, \ldots, c_{r}\right) \in \mathbb{Z}_{p_{1}}^{*} \times \cdots \times \mathbb{Z}_{p_{r}}^{*}}\left|\sum_{n=0}^{N-1} e\left(\sum_{i=1}^{r} c_{i}\left(\mathbf{h} \cdot \mathbf{z}_{n}^{(i)}\right) / p_{i}\right)\right|^{2} \\
& =\sum_{k, n=0}^{N-1}(-1)^{r} \prod_{\substack{i=1 \\
\mathbf{h} \cdot \mathbf{z}_{n}^{(i)} \equiv \mathbf{h} \cdot \mathbf{z}_{k}^{(i)}\left(\bmod p_{i}\right)}}^{r}\left(1-p_{i}\right) \\
& \leq \sum_{k, n=0}^{N-1} \prod_{\substack{i=1 \\
\mathbf{h} \cdot \mathbf{z}_{n}^{(i)} \equiv \mathbf{h} \cdot \mathbf{z}_{k}^{(i)}\left(\bmod p_{i}\right)}}^{r}\left(p_{i}-1\right) \\
& =\sum_{I \subset\{1, \ldots, r\}} \sum_{\substack{k, n=0 \\
\mathbf{h} \cdot \mathbf{z}_{n}^{(i)} \equiv \mathbf{h} \cdot \mathbf{z}_{k}^{(i)}\left(\bmod p_{i}\right), i \in I \\
\mathbf{h} \cdot \mathbf{z}_{n}^{(i)} \equiv \mathbf{h} \cdot \mathbf{z}_{k}^{(i)}\left(\bmod p_{i}\right), i \notin I}}^{N-1} \prod_{i \in I}\left(p_{i}-1\right) \\
& \leq \sum_{I \subset\{1, \ldots, r\}} \sum_{\substack{k, n=0 \\
\mathbf{h} \cdot \mathbf{z}_{n}^{(i)} \equiv \mathbf{h} \cdot \mathbf{z}_{k}^{(i)}\left(\bmod p_{i}\right), i \in I}}^{N-1} \prod_{i \in I}\left(p_{i}-1\right) \\
& =\sum_{I \subset\{1, \ldots, r\}} \sum_{k=0}^{N-1} \#\left\{0 \leq n<N \mid \mathbf{h} \cdot \mathbf{z}_{n}^{(i)} \equiv \mathbf{h} \cdot \mathbf{z}_{k}^{(i)}\left(\bmod p_{i}\right), i \in I\right\} \\
& \text {. } \prod_{i \in I}\left(p_{i}-1\right) \text {. }
\end{aligned}
$$

Now, the definition of the set $J$ implies that

$$
\begin{aligned}
\#\{0 & \left.\leq n<N \mid \mathbf{h} \cdot \mathbf{z}_{n}^{(i)} \equiv \mathbf{h} \cdot \mathbf{z}_{k}^{(i)}\left(\bmod p_{i}\right), i \in I\right\} \\
& =\#\left\{0 \leq n<N \mid \mathbf{h} \cdot \mathbf{z}_{n}^{(i)} \equiv \mathbf{h} \cdot \mathbf{z}_{k}^{(i)}\left(\bmod p_{i}\right), i \in I \backslash J\right\} \\
& \leq \#\left\{0 \leq n<m \mid \mathbf{h} \cdot \mathbf{z}_{n}^{(i)} \equiv \mathbf{h} \cdot \mathbf{z}_{k}^{(i)}\left(\bmod p_{i}\right), i \in I \backslash J\right\} \\
& =\frac{m}{m_{I \backslash J}} \#\left\{0 \leq n<m_{I \backslash J} \mid \mathbf{h} \cdot \mathbf{z}_{n}^{(i)} \equiv \mathbf{h} \cdot \mathbf{z}_{k}^{(i)}\left(\bmod p_{i}\right), i \in I \backslash J\right\} \\
& =\frac{m}{m_{I \backslash J}} \prod_{i \in I \backslash J} \#\left\{0 \leq n<p_{i} \mid \mathbf{h} \cdot \mathbf{z}_{n}^{(i)} \equiv \mathbf{h} \cdot \mathbf{z}_{k}^{(i)}\left(\bmod p_{i}\right)\right\}
\end{aligned}
$$

for $0 \leq k<N$ and $I \subset\{1, \ldots, r\}$, where in the last step the Chinese Remainder Theorem has been used. Since $\mathbf{h} \not \equiv \mathbf{0}\left(\bmod p_{i}\right)$ for $i \in\{1, \ldots, r\} \backslash J$, it follows from Lemma 4 that

$$
\#\left\{0 \leq n<p_{i} \mid \mathbf{h} \cdot \mathbf{z}_{n}^{(i)} \equiv \mathbf{h} \cdot \mathbf{z}_{k}^{(i)}\left(\bmod p_{i}\right)\right\} \leq 2 s-1
$$


for $0 \leq k<N$ and $i \in\{1, \ldots, r\} \backslash J$. Therefore,

$$
\begin{aligned}
& \sum_{\left(c_{1}, \ldots, c_{r}\right) \in \mathbb{Z}_{p_{1}}^{*} \times \cdots \times \mathbb{Z}_{p_{r}}^{*}}\left|\sum_{n=0}^{N-1} e\left(\sum_{i=1}^{r} c_{i}\left(\mathbf{h} \cdot \mathbf{z}_{n}^{(i)}\right) / p_{i}\right)\right|^{2} \\
\leq & N m \sum_{I \subset\{1, \ldots, r\}} \prod_{i \in I \backslash J} \frac{2 s-1}{p_{i}} \prod_{i \in I}\left(p_{i}-1\right) \\
= & N m \sum_{I \subset\{1, \ldots, r\}} \prod_{i \in I \backslash J} \frac{(2 s-1)\left(p_{i}-1\right)}{p_{i}} \prod_{i \in I \cap J}\left(p_{i}-1\right) \\
= & N m \prod_{i=1}^{r}\left(\frac{(2 s-1)\left(p_{i}-1\right)}{p_{i}}+1\right) \prod_{i \in J} p_{i} \\
= & N m_{J}^{2} \prod_{\substack{i \neq 1 \\
i \notin J}}^{r}\left(2 s\left(p_{i}-1\right)+1\right),
\end{aligned}
$$

which is the desired result.

Lemma 6. Let $1 \leq s<\min \left\{p_{1}, \ldots, p_{r}\right\}$ and $1 \leq N \leq 2^{-(r+1)} \prod_{i=1}^{r}\left(p_{i}-1\right)$. Then

$$
\sum_{\left(c_{1}, \ldots, c_{r}\right) \in \mathbb{Z}_{p_{1}}^{*} \times \cdots \times \mathbb{Z}_{p_{r}}^{*}}\left|\sum_{n=0}^{N-1} e\left(\sum_{i=1}^{r} c_{i} z_{s n}^{(i)} / p_{i}\right)\right|^{2}>\frac{N}{2} \prod_{i=1}^{r}\left(p_{i}-1\right) .
$$

Proof. Straightforward calculations show that

$$
\begin{aligned}
& \sum_{\left(c_{1}, \ldots, c_{r}\right) \in \mathbb{Z}_{p_{1}}^{*} \times \cdots \times \mathbb{Z}_{p_{r}}^{*}}\left|\sum_{n=0}^{N-1} e\left(\sum_{i=1}^{r} c_{i} z_{s n}^{(i)} / p_{i}\right)\right|^{2} \\
= & \sum_{I \subset\{1, \ldots, r\}}(-1)^{r-\# I} \sum_{c_{i} \in \mathbb{Z}_{p_{i}}, i \in I}\left|\sum_{n=0}^{N-1} e\left(\sum_{i \in I} c_{i} z_{s n}^{(i)} / p_{i}\right)\right|^{2} \\
= & \sum_{I \subset\{1, \ldots, r\}}(-1)^{r-\# I} \sum_{c_{i} \in \mathbb{Z}_{p_{i}}, i \in I} \sum_{k, n=0}^{N-1} \prod_{i \in I} e\left(c_{i}\left(z_{s n}^{(i)}-z_{s k}^{(i)}\right) / p_{i}\right) \\
= & \sum_{I \subset\{1, \ldots, r\}}(-1)^{r-\# I} \sum_{k, n=0}^{N-1} \prod_{i \in I} \sum_{c \in \mathbb{Z}_{p_{i}}} e\left(c\left(z_{s n}^{(i)}-z_{s k}^{(i)}\right) / p_{i}\right) \\
= & \sum_{I \subset\{1, \ldots, r\}}(-1)^{r-\# I} \sum_{k, n=0}^{N-1} m_{I} \\
= & \sum_{I \subset\{1, \ldots, r\}}(-1)^{r-\# I} m_{I} \cdot \#\left\{(k, n) \in \mathbb{Z}_{N}^{2} \mid n \equiv k\left(\bmod m_{I}\right)\right\},
\end{aligned}
$$

where $\mathbb{Z}_{N}=\{0,1, \ldots, N-1\}$. Let $N_{I} \in \mathbb{Z}_{m_{I}}$ with $N_{I} \equiv N\left(\bmod m_{I}\right)$ for subsets $I$ of $\{1, \ldots, r\}$ and observe that $m_{I}\left[N / m_{I}\right]=N-N_{I}$. Then

$$
\begin{aligned}
m_{I} \cdot \#\left\{(k, n) \in \mathbb{Z}_{N}^{2} \mid n \equiv k\left(\bmod m_{I}\right)\right\} \\
\quad=m_{I}\left(\left[N / m_{I}\right]\left(N+N_{I}\right)+N_{I}\right)=\left(N-N_{I}\right)\left(N+N_{I}\right)+m_{I} N_{I}
\end{aligned}
$$


for $I \subset\{1, \ldots, r\}$. Hence,

$$
\begin{aligned}
& \sum_{\left(c_{1}, \ldots, c_{r}\right) \in \mathbb{Z}_{p_{1}}^{*} \times \cdots \times \mathbb{Z}_{p_{r}}^{*}}\left|\sum_{n=0}^{N-1} e\left(\sum_{i=1}^{r} c_{i} z_{s n}^{(i)} / p_{i}\right)\right|^{2} \\
& =\sum_{I \subset\{1, \ldots, r\}}(-1)^{r-\# I}\left(N^{2}-N_{I}^{2}+m_{I} N_{I}\right) \\
& =N^{2} \sum_{I \subset\{1, \ldots, r\}}(-1)^{r-\# I}+\sum_{I \subset\{1, \ldots, r\}}(-1)^{r-\# I} N_{I}\left(m_{I}-N_{I}\right) \\
& =\sum_{I \subset\{1, \ldots, r\}}(-1)^{r-\# I} N_{I}\left(m_{I}-N_{I}\right) \\
& =\sum_{\substack{I \subset\{1, \ldots, r\} \\
m_{I}>N}}(-1)^{r-\# I} N\left(m_{I}-N\right)+\sum_{\substack{I \subset\{1, \ldots, r\} \\
m_{I} \leq N}}(-1)^{r-\# I} N_{I}\left(m_{I}-N_{I}\right) \\
& =\sum_{I \subset\{1, \ldots, r\}}(-1)^{r-\# I} N\left(m_{I}-N\right) \\
& +\sum_{\substack{I \subset\{1, \ldots, r\} \\
m_{I} \leq N}}(-1)^{r-\# I}\left(N_{I}\left(m_{I}-N_{I}\right)+N\left(N-m_{I}\right)\right) \\
& =N \prod_{i=1}^{r}\left(p_{i}-1\right)+\sum_{\substack{I \subset\{1, \ldots, r\} \\
m_{I} \leq N}}(-1)^{r-\# I}\left(N_{I}\left(m_{I}-N_{I}\right)+N\left(N-m_{I}\right)\right) \\
& \geq N \prod_{i=1}^{r}\left(p_{i}-1\right)-\sum_{\substack{I \subset\{1, \ldots, r\} \\
m_{I} \leq N}}\left(N_{I}\left(m_{I}-N_{I}\right)+N\left(N-m_{I}\right)\right) \\
& \geq N \prod_{i=1}^{r}\left(p_{i}-1\right)-\sum_{\substack{I \subset\{1, \ldots, r\} \\
m_{I} \leq N}}\left(N-\frac{1}{2} m_{I}\right)^{2} \\
& >N \prod_{i=1}^{r}\left(p_{i}-1\right)-2^{r} N^{2} \geq \frac{N}{2} \prod_{i=1}^{r}\left(p_{i}-1\right)
\end{aligned}
$$

for $N \leq 2^{-(r+1)} \prod_{i=1}^{r}\left(p_{i}-1\right)$.

\section{PRincipal RESUlts}

The main result of the present paper is Theorem 1, which provides an upper bound for the average value of the discrepancy of $s$-tuples in the compound inversive congruential method over the parameters $\left(c_{1}, \ldots, c_{r}\right) \in \mathbb{Z}_{p_{1}}^{*} \times \cdots \times \mathbb{Z}_{p_{r}}^{*}$. Theorem 2 is an immediate consequence of this result. A proof is added for the sake of completeness. In Theorem 3 a corresponding lower bound for the discrepancy of $s$-tuples is established.

Theorem 1. Let $1 \leq s<\min \left\{p_{1}, \ldots, p_{r}\right\}, 1 \leq N \leq m$, and $a_{i} \in \mathbb{M}_{p_{i}}$ for $1 \leq i \leq$ $r$. Then the average value of the discrepancy $D_{N ; c_{1}, \ldots, c_{r}}^{(s)}$ of $s$-tuples in the compound 
inversive congruential method over $\left(c_{1}, \ldots, c_{r}\right) \in \mathbb{Z}_{p_{1}}^{*} \times \cdots \times \mathbb{Z}_{p_{r}}^{*}$ satisfies

$$
\begin{aligned}
& \frac{1}{\prod_{i=1}^{r}\left(p_{i}-1\right)} \sum_{\left(c_{1}, \ldots, c_{r}\right) \in \mathbb{Z}_{p_{1}}^{*} \times \cdots \times \mathbb{Z}_{p_{r}}^{*}} D_{N ; c_{1}, \ldots, c_{r}}^{(s)} \\
& \quad<(\sqrt{2 s+0.25}+0.5)^{r} N^{-1 / 2}\left(\frac{2}{\pi} \log m+\frac{7}{5}\right)^{s} .
\end{aligned}
$$

Proof. First, Lemma 1 is applied with $k=s, q=m$, and $\mathbf{t}_{n}=\mathbf{x}_{n}$ for $0 \leq n<N$. This yields

$$
\begin{aligned}
D_{N ; c_{1}, \ldots, c_{r}}^{(s)} & \leq \frac{s}{m}+\frac{1}{N} \sum_{\mathbf{h} \in C_{s}(m)} \frac{1}{r(\mathbf{h}, m)}\left|\sum_{n=0}^{N-1} e\left(\mathbf{h} \cdot \mathbf{x}_{n}\right)\right| \\
& =\frac{s}{m}+\frac{1}{N} \sum_{\mathbf{h} \in C_{s}(m)} \frac{1}{r(\mathbf{h}, m)}\left|\sum_{n=0}^{N-1} e\left(\sum_{i=1}^{r} c_{i}\left(\mathbf{h} \cdot \mathbf{z}_{n}^{(i)}\right) / p_{i}\right)\right|
\end{aligned}
$$

for any $\left(c_{1}, \ldots, c_{r}\right) \in \mathbb{Z}_{p_{1}}^{*} \times \cdots \times \mathbb{Z}_{p_{r}}^{*}$, where the $s$-tuple $\mathbf{z}_{n}^{(i)}$ is defined as in the second section. Therefore, the average value of the discrepancy $D_{N ; c_{1}, \ldots, c_{r}}^{(s)}$ over $\left(c_{1}, \ldots, c_{r}\right) \in \mathbb{Z}_{p_{1}}^{*} \times \cdots \times \mathbb{Z}_{p_{r}}^{*}$ satisfies

$$
\begin{aligned}
& \frac{1}{\prod_{i=1}^{r}\left(p_{i}-1\right)} \sum_{\left(c_{1}, \ldots, c_{r}\right) \in \mathbb{Z}_{p_{1}}^{*} \times \cdots \times \mathbb{Z}_{p_{r}}^{*}} D_{N ; c_{1}, \ldots, c_{r}}^{(s)} \leq \frac{s}{m}+\frac{1}{N} \sum_{\mathbf{h} \in C_{s}(m)} \frac{1}{r(\mathbf{h}, m)} \\
& \cdot\left(\frac{1}{\prod_{i=1}^{r}\left(p_{i}-1\right)} \sum_{\left(c_{1}, \ldots, c_{r}\right) \in \mathbb{Z}_{p_{1}}^{*} \times \cdots \times \mathbb{Z}_{p_{r}}^{*}}\left|\sum_{n=0}^{N-1} e\left(\sum_{i=1}^{r} c_{i}\left(\mathbf{h} \cdot \mathbf{z}_{n}^{(i)}\right) / p_{i}\right)\right|\right) \\
& \leq \frac{s}{m}+\frac{1}{N} \sum_{\mathbf{h} \in C_{s}(m)} \frac{1}{r(\mathbf{h}, m)} \\
& \cdot \sqrt{\frac{1}{\prod_{i=1}^{r}\left(p_{i}-1\right)} \sum_{\left(c_{1}, \ldots, c_{r}\right) \in \mathbb{Z}_{p_{1}}^{*} \times \cdots \times \mathbb{Z}_{p_{r}}^{*}}\left|\sum_{n=0}^{N-1} e\left(\sum_{i=1}^{r} c_{i}\left(\mathbf{h} \cdot \mathbf{z}_{n}^{(i)}\right) / p_{i}\right)\right|^{2}} \\
& =\frac{s}{m}+\frac{1}{N} \sum_{\substack{J \subset\{1, \ldots, r\} \\
\# J<r}} \sum_{\substack{\left.\mathbf{h} \in \mathbf{h} \in C_{s}(m) \\
\mathbf{h} \neq \mathbf{m o d} p_{i}\right), i \in J}} \frac{1}{r(\mathbf{h}, m)} \\
& \cdot \sqrt{\frac{1}{\prod_{i=1}^{r}\left(p_{i}-1\right)} \sum_{\left(c_{1}, \ldots, c_{r}\right) \in \mathbb{Z}_{p_{1}}^{*} \times \cdots \times \mathbb{Z}_{p_{r}}^{*}}\left|\sum_{n=0}^{N-1} e\left(\sum_{i=1}^{r} c_{i}\left(\mathbf{h} \cdot \mathbf{z}_{n}^{(i)}\right) / p_{i}\right)\right|^{2}},
\end{aligned}
$$

where the penultimate step follows from Schwarz's inequality. Now, Lemma 5 can be used in order to obtain 


$$
\begin{gathered}
\frac{1}{\prod_{i=1}^{r}\left(p_{i}-1\right)} \sum_{\substack{\left(c_{1}, \ldots, c_{r}\right) \in \mathbb{Z}_{p_{1}}^{*} \times \cdots \times \mathbb{Z}_{p_{r}}^{*} \\
\leq}} D_{N ; c_{1}, \ldots, c_{r}}^{(s)} \sum_{\substack{J \subset\{1, \ldots, r\} \\
\# J<r}} \sum_{\substack{\mathbf{h} \in C_{s}(m) \\
\mathbf{h}=\mathbf{0}\left(\bmod p_{i}\right), i \in J \\
\mathbf{h} \neq \mathbf{0}\left(\bmod p_{i}\right), i \notin J}} \frac{1}{r(\mathbf{h}, m)} \\
\cdot \sqrt{\sum_{\substack{i=1 \\
i \notin J}}^{N m_{J}^{2} \prod^{r}\left(2 s+\left(p_{i}-1\right)^{-1}\right) \prod_{i \in J}\left(p_{i}-1\right)^{-1}}} \\
\leq \frac{s}{m}+\frac{1}{N^{1 / 2}} \sum_{\substack{J \subset\{1, \ldots, r\} \\
\# J<r}} \prod_{\substack{i=1 \\
i \notin J}}^{r}\left(2 s+\left(p_{i}-1\right)^{-1}\right)^{1 / 2} \\
\cdot \prod_{i \in J}\left(p_{i}-1\right)^{-1 / 2} m_{J} \sum_{\substack{\mathbf{h} \in C_{s}(m) \\
\mathbf{h} \equiv \mathbf{0}\left(\bmod m_{J}\right)}} \frac{1}{r(\mathbf{h}, m)},
\end{gathered}
$$

where $m_{J}=\prod_{i \in J} p_{i}$ for subsets $J$ of $\{1, \ldots, r\}$. Hence, it follows from Lemma 3 that

$$
\begin{aligned}
& \frac{1}{\prod_{i=1}^{r}\left(p_{i}-1\right)} \sum_{\substack{\left(c_{1}, \ldots, c_{r}\right) \in \mathbb{Z}_{p_{1}}^{*} \times \cdots \times \mathbb{Z}_{p_{r}}^{*} \\
<}} D_{N ; c_{1}, \ldots, c_{r}}^{(s)}+\frac{1}{N^{1 / 2}} \sum_{\substack{J \subset\{1, \ldots, r\} \\
\# J<r}} \prod_{\substack{i=1 \\
i \notin J}}^{r}\left(2 s+\left(p_{i}-1\right)^{-1}\right)^{1 / 2} \\
& \cdot \prod_{i \in J}\left(p_{i}-1\right)^{-1 / 2}\left(\frac{2}{\pi} \log m+\frac{7}{5}\right)^{s} \\
&< \frac{1}{N^{1 / 2}} \sum_{J \subset\{1, \ldots, r\}} \prod_{i=1}^{r}\left(2 s+\left(p_{i}-1\right)^{-1}\right)^{1 / 2} \\
& \cdot \prod_{i \in J}\left(p_{i}-1\right)^{-1 / 2}\left(\frac{2}{\pi} \log m+\frac{7}{5}\right)^{s} \\
&= \frac{1}{N^{1 / 2}} \prod_{i=1}^{r}\left(\left(2 s+\left(p_{i}-1\right)^{-1}\right)^{1 / 2}+\left(p_{i}-1\right)^{-1 / 2}\right)\left(\frac{2}{\pi} \log m+\frac{7}{5}\right)^{s} \\
& \leq \frac{1}{N^{1 / 2}}\left((2 s+0.25)^{1 / 2}+0.5\right)^{r}\left(\frac{2}{\pi} \log m+\frac{7}{5}\right)^{s},
\end{aligned}
$$

which is the desired result.

Theorem 2. Let $1 \leq s<\min \left\{p_{1}, \ldots, p_{r}\right\}, 1 \leq N \leq m$, and $a_{i} \in \mathbb{M}_{p_{i}}$ for $1 \leq i \leq r$ be fixed. Let $0<\alpha \leq 1$. Then there exist more than $(1-\alpha) \prod_{i=1}^{r}\left(p_{i}-1\right)$ values of $\left(c_{1}, \ldots, c_{r}\right) \in \mathbb{Z}_{p_{1}}^{*} \times \cdots \times \mathbb{Z}_{p_{r}}^{*}$ such that the discrepancy $D_{N ; c_{1}, \ldots, c_{r}}^{(s)}$ of s-tuples in the 
compound inversive congruential method satisfies

$$
D_{N ; c_{1}, \ldots, c_{r}}^{(s)}<\frac{1}{\alpha}(\sqrt{2 s+0.25}+0.5)^{r} N^{-1 / 2}\left(\frac{2}{\pi} \log m+\frac{7}{5}\right)^{s} .
$$

Proof. Subsequently, the abbreviation

$$
M=(\sqrt{2 s+0.25}+0.5)^{r} N^{-1 / 2}\left(\frac{2}{\pi} \log m+\frac{7}{5}\right)^{s}
$$

is used. Suppose that there exist at most $(1-\alpha) \prod_{i=1}^{r}\left(p_{i}-1\right)$ values of $\left(c_{1}, \ldots, c_{r}\right) \in$ $\mathbb{Z}_{p_{1}}^{*} \times \cdots \times \mathbb{Z}_{p_{r}}^{*}$ with $D_{N ; c_{1}, \ldots, c_{r}}^{(s)}<\alpha^{-1} M$, i.e., there exist at least $\alpha \prod_{i=1}^{r}\left(p_{i}-1\right)$ values of $\left(c_{1}, \ldots, c_{r}\right) \in \mathbb{Z}_{p_{1}}^{*} \times \cdots \times \mathbb{Z}_{p_{r}}^{*}$ with $D_{N ; c_{1}, \ldots, c_{r}}^{(s)} \geq \alpha^{-1} M$. Hence, one obtains

$$
\sum_{\left(c_{1}, \ldots, c_{r}\right) \in \mathbb{Z}_{p_{1}}^{*} \times \cdots \times \mathbb{Z}_{p_{r}}^{*}} D_{N ; c_{1}, \ldots, c_{r}}^{(s)} \geq M \prod_{i=1}^{r}\left(p_{i}-1\right),
$$

which contradicts Theorem 1.

Theorem 3. Let $1 \leq s<\min \left\{p_{1}, \ldots, p_{r}\right\}, 1 \leq N \leq 2^{-(r+1)} \prod_{i=1}^{r}\left(p_{i}-1\right)$, and $a_{i} \in \mathbb{M}_{p_{i}}$ for $1 \leq i \leq r$ be fixed. Then there exist parameters $\left(c_{1}, \ldots, c_{r}\right) \in$ $\mathbb{Z}_{p_{1}}^{*} \times \cdots \times \mathbb{Z}_{p_{r}}^{*}$ such that the discrepancy $D_{N ; c_{1}, \ldots, c_{r}}^{(s)}$ of $s$-tuples in the compound inversive congruential method satisfies

$$
D_{N ; c_{1}, \ldots, c_{r}}^{(s)}>\frac{1}{2 \sqrt{2}} N^{-1 / 2} .
$$

Proof. First, Lemma 2 is applied with $k=s, \mathbf{t}_{n}=\mathbf{x}_{n}$ for $0 \leq n<N$, and $\mathbf{h}=(1,0, \ldots, 0) \in \mathbb{Z}^{s}$. This yields

$$
D_{N ; c_{1}, \ldots, c_{r}}^{(s)} \geq \frac{1}{2 N}\left|\sum_{n=0}^{N-1} e\left(\sum_{i=1}^{r} c_{i} z_{s n}^{(i)} / p_{i}\right)\right| .
$$

Now, the desired result follows at once from Lemma 6.

\section{Discussion}

First, it should be observed that the main results apply for the full period $(N=m)$ as well as for parts of the period $(N<m)$, for equidistribution properties $(s=1)$ as well as for statistical independence properties $(s \geq 2)$, and for the ordinary inversive congruential method $(r=1)$ as well as for the compound method $(r \geq 2)$. In the following, let the number $r$ of prime factors of $m$ be fixed. Then Theorem 1 shows that for any parameters $a_{1} \in \mathbb{M}_{p_{1}}, \ldots, a_{r} \in \mathbb{M}_{p_{r}}$ in the compound inversive congruential method and any dimension $s$ the discrepancy $D_{N ; c_{1}, \ldots, c_{r}}^{(s)}$, on the average over the parameters $c_{1}, \ldots, c_{r}$, has an order of magnitude at most $N^{-1 / 2}(\log m)^{s}$. It should be observed that this upper bound is independent of the specific choice of the parameters $a_{1}, \ldots, a_{r}$. This result is basically in accordance with the law of the iterated logarithm for the discrepancy of $N$ true random points from $[0,1)^{s}$, which is almost always of an order of magnitude $N^{-1 / 2}(\log \log N)^{1 / 2}$ (cf. [9]). Theorem 2 provides even more information, since it implies that for any parameters $a_{1}, \ldots, a_{r}$ and any dimension $s$ only an arbitrarily small percentage of the parameters $c_{1}, \ldots, c_{r}$ may lead to a discrepancy $D_{N ; c_{1}, \ldots, c_{r}}^{(s)}$ with an order of magnitude greater than $N^{-1 / 2}(\log m)^{s}$. On the other hand, Theorem 3 shows that for any parameters $a_{1}, \ldots, a_{r}$ and any dimension $s$ there exist parameters $c_{1}, \ldots, c_{r}$ 
such that the discrepancy $D_{N ; c_{1}, \ldots, c_{r}}^{(s)}$ is of an order of magnitude at least $N^{-1 / 2}$, provided $N$ is not too large.

\section{REFERENCES}

1. J. Eichenauer-Herrmann, Inversive congruential pseudorandom numbers avoid the planes, Math. Comp. 56 (1991), 297-301. MR 91k:65021

2. _ Inversive congruential pseudorandom numbers: a tutorial, Internat. Statist. Rev. 60 (1992), 167-176.

3. _ Improved lower bounds for the discrepancy of inversive congruential pseudorandom numbers, Math. Comp. 62 (1994), 783-786. MR 94g:11058

4. _ On generalized inversive congruential pseudorandom numbers, Math. Comp. 63 (1994), 293-299. MR 94k:11088

5. (1995), 247-255.

6. and their Appl. 1 (1995), 102-114.

7. M. Flahive and H. Niederreiter, On inversive congruential generators for pseudorandom numbers, Finite Fields, Coding Theory, and Advances in Communications and Computing (G. L. Mullen and P. J.-S. Shiue, eds.), Dekker, New York, 1993, pp. 75-80. MR 94a:11117

8. K. Huber, On the period length of generalized inversive pseudorandom number generators, Appl. Algebra Engrg. Comm. Comput. 5 (1994), 255-260. CMP: 9414

9. J. Kiefer, On large deviations of the empiric d.f. of vector chance variables and a law of the iterated logarithm, Pacific J. Math. 11 (1961), 649-660. MR 24:A1732

10. H. Niederreiter, The serial test for congruential pseudorandom numbers generated by inversions, Math. Comp. 52 (1989), 135-144. MR 90e:65008

11. L_ Lower bounds for the discrepancy of inversive congruential pseudorandom numbers, Math. Comp. 55 (1990), 277-287. MR 91e:65016

12. _ Recent trends in random number and random vector generation, Ann. Oper. Res. 31 (1991), 323-345. MR 92h:65010

13. Theory, and Advances in Communications and Computing (G. L. Mullen and P. J.-S. Shiue, eds.), Dekker, New York, 1993, pp. 375-394. MR 94a:11121

14. Nonlinear methods for pseudorandom number and vector generation, Simulation and Optimization (G. Pflug and U. Dieter, eds.), Lecture Notes in Econom. and Math. Systems, vol. 374, Springer, Berlin, 1992, pp. 145-153.

15. - Random number generation and quasi-Monte Carlo methods, SIAM, Philadelphia, PA, 1992. MR 93h:65008

Fachbereich Mathematik, Technische Hochschule Darmstadt, SchlossgartenStrasse 7, D-64289 Darmstadt, Germany 